\title{
Assessing the contribution of different uncertainty sources in streamflow projections
}

\begin{abstract}
Hydrological models are commonly used to quantify the hydrological impacts of climate change using general circulation model (GCM) simulations as input. However, application of the model results with respect to future changes in streamflow scenarios remains limited by the large uncertainties stemming from various sources. Therefore, this study aimed to explore uncertainties involved in climate change impact assessment in Hulu Langat Basin, Malaysia, and define the contribution of uncertainty sources to the final uncertainty level. Hydrological model parameters, GCMs, and emission scenario uncertainties were considered the main uncertainty contributors in local-scale impact studies. The equidistant quantile matching method is used to bias-correct simulations of 19 GCMs under two emission scenarios of RCP4.5 and RCP8.5. The Soil and Water Assessment Tool (SWAT) hydrological model is next run by the bias-corrected GCM data to generate a wide spectrum of future streamflow scenarios. Projected monthly streamflow pattern under RCP8.5 showed a different temporal pattern from the observed one. Hydrological model parameter uncertainty was proven to be a larger uncertainty contributor than emission scenario during baseline climate. GCM and emission scenario uncertainties escalated as progressed in time and GCM uncertainty showed larger increments. The monthly pattern of effect of each uncertainty source varied when comparing the two periods of 2030s and 2080s. Therefore, for a superior management of water resources, a study of climate change impacts and uncertainty sources on a smaller scale than the decadal or annual scales can be more informative to the decision makers.
\end{abstract}

